\title{
Synthesis of Conductive Ink Based on Silver Nanoparticles and its Application in Inkjet Printing Patterns on Glass Substrates
}

\author{
Chien Mau Dang ${ }^{1}$, Huong Kim Tran², Dung My Thi Dang ${ }^{3}$ \\ ${ }^{1,2,3}$ Institute for Nanotechnology, Vietnam National University - Ho Chi Minh City, Community 6, Linh Trung Ward, Thu Duc \\ District, Ho Chi Minh City, Vietnam
}

\begin{abstract}
This paper presents a synthesis of silver nanoparticles-based solution which was then used as conductive ink for inkjet printing application. In this work, colloidal silver nanoparticles (AgNPs) were synthesized from silver nitrate $\left(\mathrm{AgNO}_{3}\right)$ with polyvinyl pyrrolidone (PVP) as a capping agent and ethylene glycol (EG) as a solvent and reducing agent. After the synthesis, the silver nanoparticle solution was characterized by Ultraviolet-visible (UV-Vis) spectrophotometry Fourier transform infrared (FTIR) method. Transmission electron microscopy (TEM) showed the nanoparticles diameter ranged from $10 \mathrm{~nm}$ to $60 \mathrm{~nm}$ which is suitable for printing process through the nozzles of the inkjet printhead (the diameter of the printing nozzles was $21.5 \mu \mathrm{m}$ ). After that, some samples with a square shape were printed using this conductive ink on glass substrates which were then heated at different temperatures by a hot plate. The samples were characterized by Field emission-Scanning electron microscopy (FE-SEM) and a non-contact surface charge analyzer. It was found that with the heat-treatment from $200^{\circ} \mathrm{C}$ to $250^{\circ} \mathrm{C}$ the printed samples obtained the resistivity of 0.4-0.5 $\Omega$, which could be used for electronic applications.
\end{abstract}

KEYWORDS: AgNPs, Silver nanoparticles, conductive ink, inkjet printing

\section{INTRODUCTION}

Nowadays, Silver Nanoparticles (AgNPs) have attracted much interest due to their shape and size-dependent properties, which lead to variety in their properties such as mechanical property, catalytic activity, thermal and electrical conductivity, optical absorption and melting point [1-3]. Therefore, the AgNPs have been being used for many applications such as antibacterial activity [4-6], optical sensor [7], components in composite materials [8], integrated circuits [9] and others [10-11]. In addition, there have been variety methods to synthesize the AgNPs such as chemical reduction [12-14], microwave assisted method [15, 16], irradiation methods $[17,18] \ldots$ According to statistics from the related references, the chemical reduction is the most common approach for the synthesis of the AgNPs [19]. Chemical reduction is a simple method and can control particle size easily. In reduction reaction, $\mathrm{Ag}^{+}$ions are reduced into $\mathrm{Ag}$ atoms: $\mathrm{Ag}^{+}+\mathrm{e}-\rightarrow \mathrm{Ag}^{0}$. These $\mathrm{Ag}$ atoms formed during the reduction process can collide with metal ions in the solution. Thus, a reduction agent can greatly affect the nanoparticle formation. Particle size is dependent on reduction property of the reduction agents so these agents need to be as strong as possible in order to obtain the small nanoparticle sizes. Moreover, to disperse the nanoparticles well in solvents and avoid them from agglomeration, several polymers have been used as capping agents. Choosing a suitable polymer which can influence agglomeration process of the AgNPs and can easily be eliminated through centrifugation is very important. Polyvinyl pyrrolidone (PVP) has been used thanks to its' excellent characteristics including stability, controlled particle size, protective capacity of the AgNPs to prevent their agglomeration and oxidation processes and ability to be decomposed after centrifuging and sintering [18, 20-21].

The centrifuge method is a popular method to obtain the metal nanoparticles in a solution. In rotating centrifuge, a centrifugal force will cause the particles in the tubes to settle to the bottom. The settling rate depends on the centrifugal force, the density of the particles, the viscosity of the solution, the size and shape of the particles. To completely settle the metal nanoparticles, an optimal ratio between the AgNPs colloid solution and the centrifugal solvent should be studied and established. After washed by centrifugation, the AgNPs are deposited at the bottom of the tube, the upper colorless solution is removed and the nanoparticles can be collected afterward.

In this study, we presented a synthesis process of the AgNPs using PVP as a surfactant and EG as a reducing agent by the ultrasonic stirring method. This method was simple, easily performed and could reduce the reaction time. After underwent the centrifugation, the AgNPs were subsequently dispersed in several solvents such as ethanol, isopropanol, acetone and glycerol to form conductive ink for inkjet printing test. The AgNPs were characterized with UVVis absorption spectroscopy, Fourier transform infrared 
(FTIR) spectroscopy, field emission-scanning electron microscopy (FE-SEM), transmission electron microscopy (TEM). The conductive ink was used for inkjet printing and the printed samples were characterized with a Surface charge analyzer to measure resistivity of the samples.

\section{EXPERIMENTAL}

\subsection{Materials}

The chemicals used for the experiments included silver nitrate $\left(\mathrm{AgNO}_{3}\right)$ purchased from Merck, Germany, polyvinyl pyrrolidone (PVP, average molecular weight of 10,000) purchased from Sigma-Aldrich. The solvents such as ethanol, isopropanol, ethylene glycol (EG), glycerol were purchased from Merck, Germany, acetone was purchased from China.

\subsection{Synthesis of silver nanoparticles}

Firstly, $1.6 \mathrm{~g}$ PVP was dissolved in $40 \mathrm{ml}$ EG by an ultrasonic probe and this solution was cooled down to room temperature. Next, $8.84 \mathrm{~g} \mathrm{AgNO}_{3}$ was added into PVP/EG solution. An ultrasonic probe (VCX 750, Sonics, USA) was immersed into the above solution for 3 minutes 10 seconds. At the beginning of the reaction, the yellowish-colored solution turned darker, indicating the formation of AgNPs. This reaction ended when the solution had darker greencolor. Afterwards, the ultra-centrifuge system (CP80NX, Hitachi, Japan) was used to wash and precipitate the AgNPs. For the first time, acetone was added into the above solution and the ultra-centrifuge system was used with $10,000 \mathrm{rpm}$ until the acetone color became colorless. For the second time, deionization (DI) water and acetone (the volume ratio between water and acetone was 1:3) were also added to the solution to wash and collect AgNPs powders when using the ultra-centrifuge system (with $15,000 \mathrm{rpm})$.

\subsection{Preparation of the conductive solution in organic} solvents

The AgNPs powders were dispersed in $2.28 \mathrm{ml}$ of several solvents such as $20 \mathrm{wt} \%$ DI, $22 \mathrm{wt} \%$ ethanol, $3 \mathrm{wt} \%$ ethylene glycol, $5 \mathrm{wt} \%$ isopropanol, $40 \mathrm{wt} \%$ glycerol to prepare the $10 \mathrm{wt} \%$ conductive solution. These solvents are some common solvents to fabricate ink in many researches. Finally, the conductive ink solution was printed on the glass substrates by the inkjet printer DMP-2800, Fujifilm Dimatix, USA.

\subsection{Inkjet printing experiments}

The inkjet printer DMP-2800 (Fujifilm Dimatix, USA) contained a cartridge which had 16 nozzles of 10-picoliter drop volume (DMCLCP-11610). The parameters of the inkjet printing process included substrate temperature set at $30{ }^{\circ} \mathrm{C}$, drop spacing of $15 \mu \mathrm{m}$ and voltage of $22 \mathrm{~V}$. These parameters were optimized conditions to obtain good quality of the printed lines and the ink droplets. The printer was used for printing a square with dimension of $10 \mathrm{~mm} \times 10$ $\mathrm{mm}$ on a glass substrate. After printing, the samples were heated at various temperatures $\left(100^{\circ} \mathrm{C}, 150^{\circ} \mathrm{C}, 200^{\circ} \mathrm{C}\right.$ and $\left.250^{\circ} \mathrm{C}\right)$.

\subsection{Characterization}

The AgNPs in water was studied by UV-Vis adsorption spectroscopy with a double-beam spectrophotometer (Varian Cary 100) in the wavelength range from 350 to $650 \mathrm{~nm}$ to identify the formation of the AgNPs by the plasmon peak. Moreover, Fourier transform infrared spectrometer (Bruker, Lumos) using Attenuated Total Reflection - ATR sampling mode was used to investigate the function of the capping agent PVP protecting the AgNPs. This mode was a measurement technique of FTIR which required a good contact between ATR crystal and the sample.

The morphology and size of the AgNPs in the conductive ink solution were observed by transmission electron microscopy (TEM, JEM-2100F, JEOL, Japan).

The surface morphology of the conductive silver patterns heated at different temperatures after printing was observed by field emission-scanning electron microscopy (FE-SEM, SU8010, Hitachi, Japan). Moreover, the influence of heat treatment on the resistivity of the conductive patterns was investigated with the surface charge analyzer (EddyCus TF map 2525 SR, Germany).

\section{RESULTS AND DISCUSSION}

\subsection{The formation of silver nanoparticles}

After the AgNPs colloids synthesis, the solution was analyzed by UV-Vis spectrometer and the result was shown in Figure 1. Figure 1 showed the optical absorbance which appeared at a wavelength between $400 \mathrm{~nm}$ and $420 \mathrm{~nm}$. This was attributed to the surface plasmon resonance (SPR) peak of the silver nanoparticles [22]. 


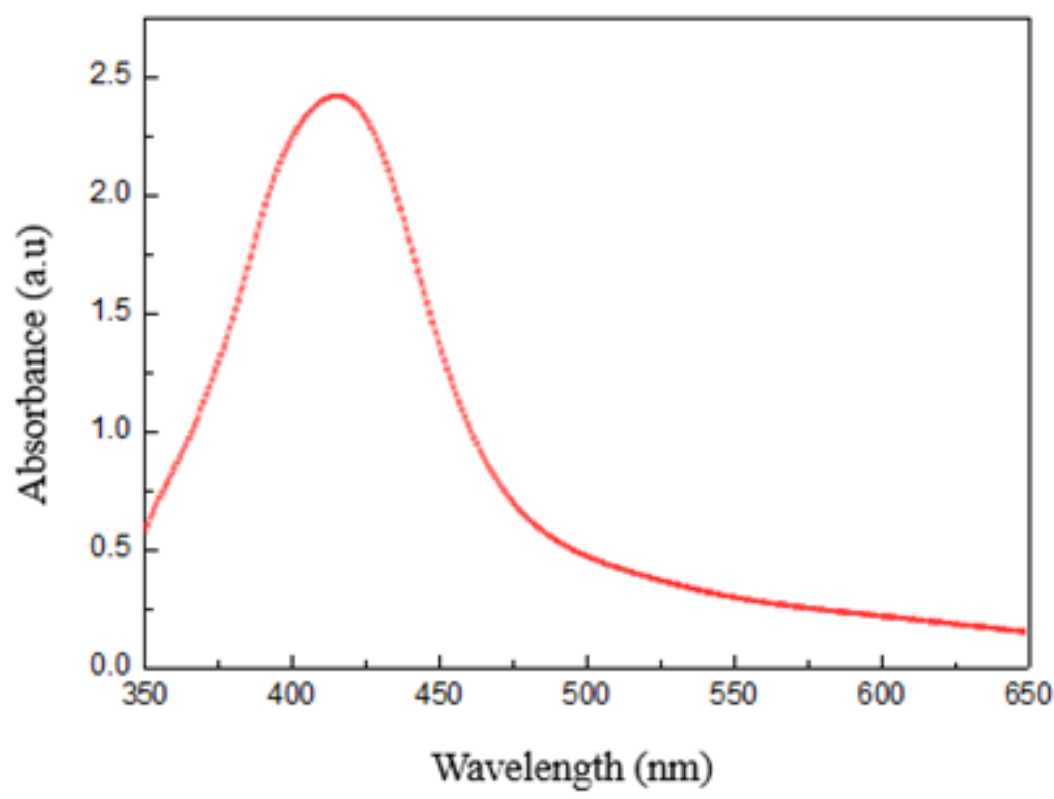

Figure 1. The UV-Vis spectra of the AgNPs with PVP in the EG solution.

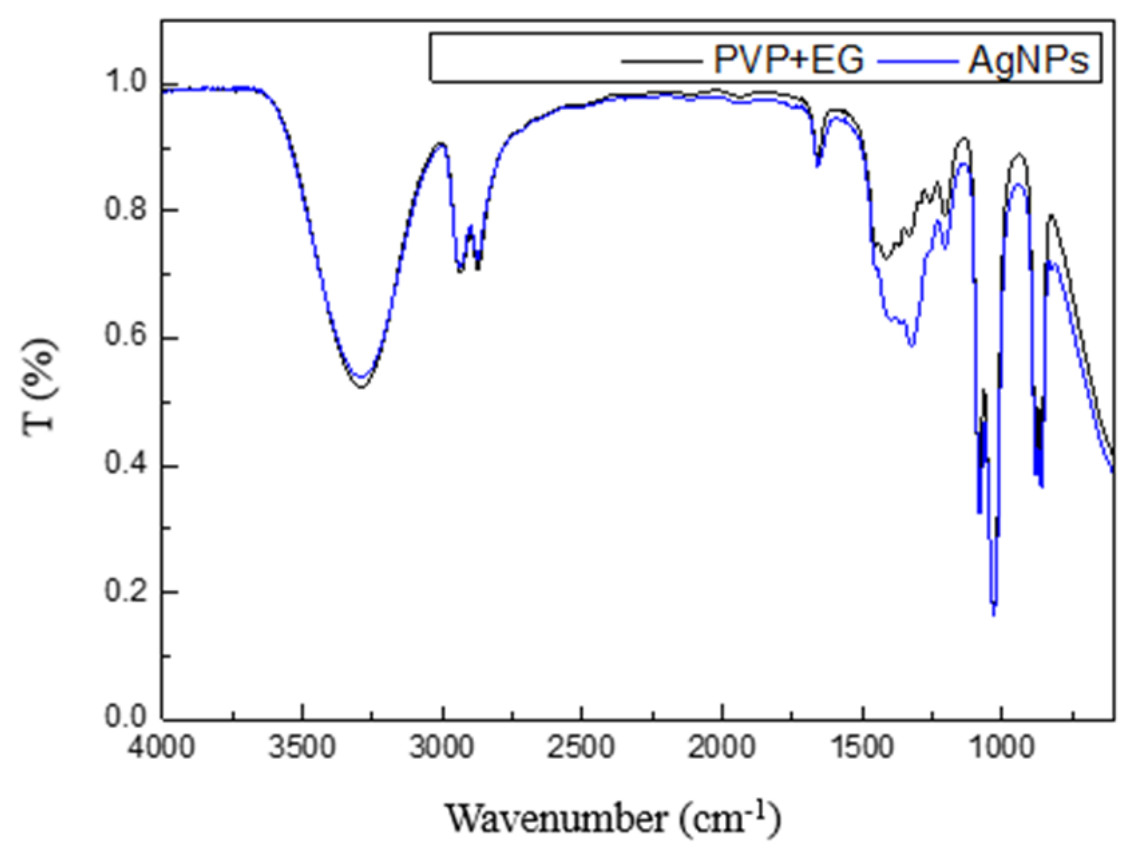

Figure 2. FTIR spectrum of PVP + EG (black) and FTIR spectrum of AgNPs (blue).

Figure 2 showed FTIR spectra of PVP+EG (black color) and the synthesized AgNPs (blue). It can be seen that both spectra are quite similar. However, the FTIR spectrum (black) had a small absorbent peak of $\mathrm{C}=\mathrm{O}$ at $1245 \mathrm{~cm}^{-1}$ which featured of pyrolodone in PVP. The FTIR spectrum (blue) appeared a peak $1322 \mathrm{~cm}^{-1}$ of $\mathrm{C}-\mathrm{O}$ but the evanescence of the peaks at $1245 \mathrm{~cm}^{-1}$ and the two peaks of $\mathrm{C}-\mathrm{N}$ at $1030 \mathrm{~cm}^{-1}, 1088 \mathrm{~cm}^{-1}$ were enhanced. It proved that the particles grew up and prevented $\mathrm{C}=\mathrm{O}$ in the pyrrolodone group and N in PVP coordinated with the silver.

\subsection{The formulation of the conductive ink solution}

Furthermore, the AgNPs had spherical shape as shown in Figure 3 imaged by transmission electron microscopy (TEM). Figure 3 indicated that the majority of the AgNPs size was in the range of 10- $60 \mathrm{~nm}$ and they had spherical shape. Figure 3 also showed that the nanoparticles were kept separated probably due to the fact that the surface of the AgNPs was fully coated with PVP. 

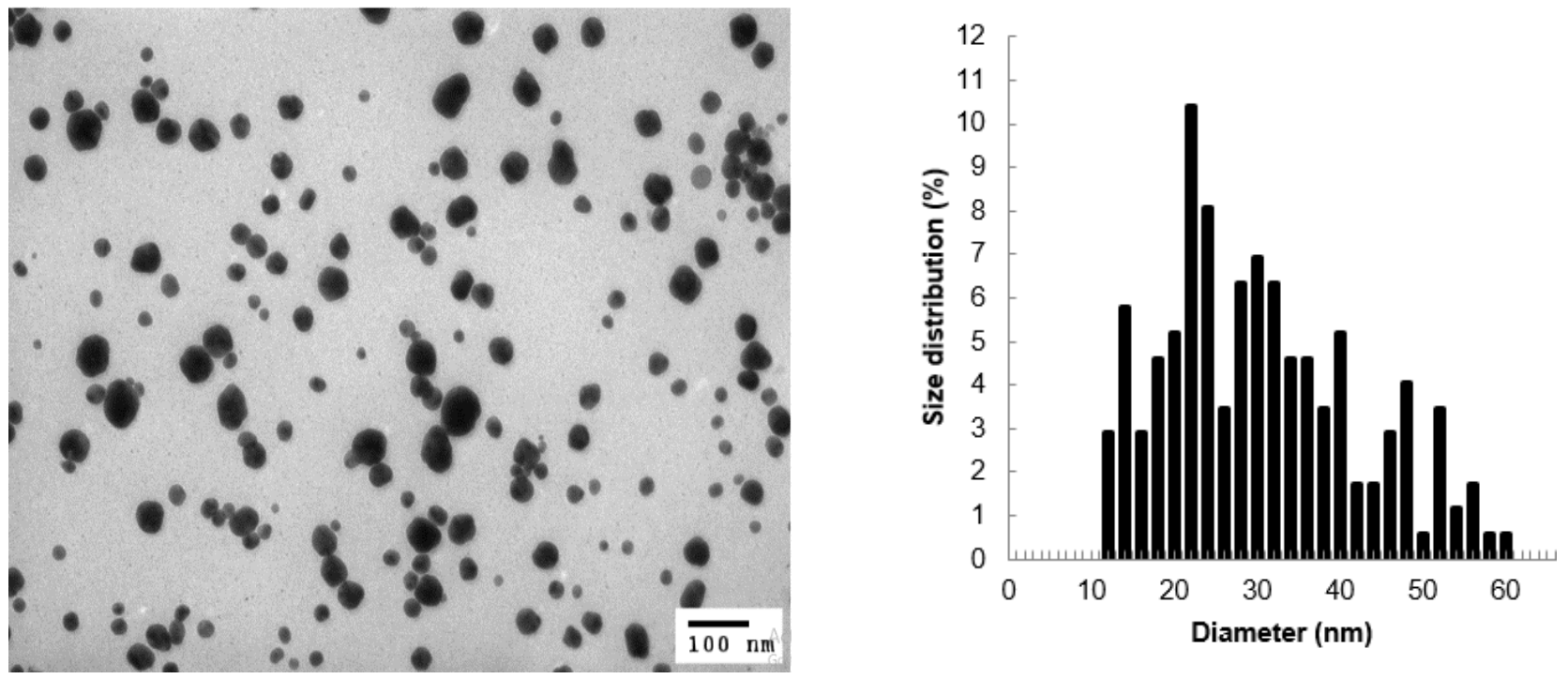

Figure 3. TEM image and size distribution of the AgNPs in ethylene glycol.

\subsection{Influence of heating temperature on the printed conductive pattern}

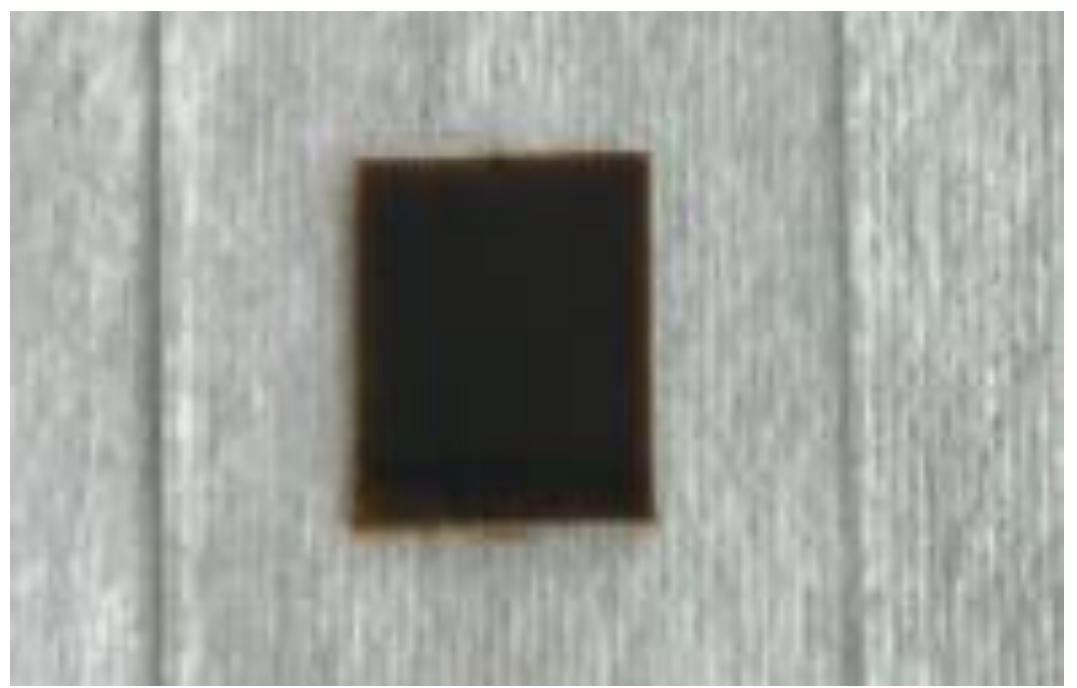

Figure 4. The square sample $(10 \times 10 \mathrm{~mm})$ was printed with the conductive ink solution.

After the printing process, the heating temperature was an important factor which affected the resistivity of the conductive silver pattern. The heating process helped to remove the solvents and the capping agent in the conductive solution. During heat-treatment process, the nanoparticles also became aggregated and its size also increased.

At different temperatures of heating the conductive patterns obtained different resistivity values and it required the patterns had to be conductive. At temperature of $100^{\circ} \mathrm{C}$, the nanoparticles were dispersed and the distance of them was quite far (Figure 5a). When the heating temperature increased, the particles size also increased. It was observed obviously at $150^{\circ} \mathrm{C}$ the particles were melted, which led to increased size (Figure 5b). With the heat treatment at $200^{\circ} \mathrm{C}$, the nanoparticles started to adhere together, the distance between two particles was shortened and the particles agglomerated (Figure 5c). Figure 5 also showed that the AgNPs microstructure had the tendency to form a constant film when the heat-treatment temperature was increasing. By $200^{\circ} \mathrm{C}$, AgNPs were melted and sintered. At $250^{\circ} \mathrm{C}$, the significant change of the particle shape and size was observed because of the melted and sintered particles (Figure 5d). Figure 5d showed the nanoparticles were sintered to form some large clusters. 

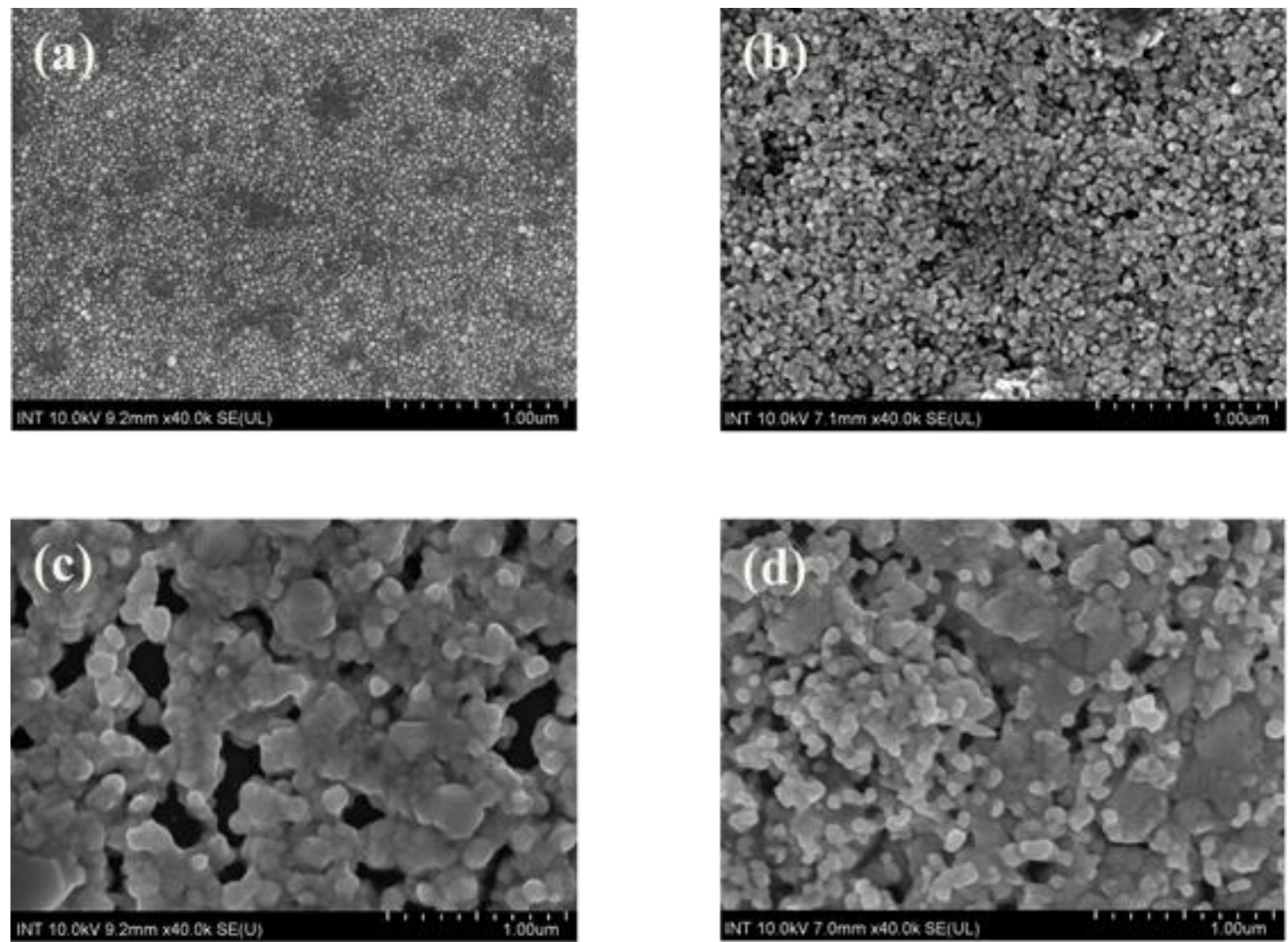

Figure 5. FESEM images of the conductive pattern corresponding with the heating temperature of (a) $100^{\circ} \mathrm{C},(\mathrm{b}) 150^{\circ} \mathrm{C},(\mathrm{c})$ $200^{\circ} \mathrm{C}$ and (d) $250^{\circ} \mathrm{C}$.

In addition, to investigate the conductivity of these films, the films were measured with the non-contact surface charge analyzer (EddyCus TF map 2525 SR, Germany) and the results of resitivity were shown in Table 1 . It could be seen that when the temperature increased the resistivity of the films decreased. The resistivity measurement results fully complied with the results of FE-SEM investigations with the above explanations. At temperature of above $200^{\circ} \mathrm{C}$, the resistivity of the films was almost unchanged. Therefore, the heat-treatment from $200^{\circ} \mathrm{C}$ to $250^{\circ} \mathrm{C}$ was suitable temperature to obtain the films with good conductivity.

Table 1. The resistivity of the films with various heat-treatment temperatures.

\begin{tabular}{|l|l|}
\hline Temperature $\left({ }^{\mathbf{0}} \mathbf{C}\right)$ & Resistivity $(\mathbf{\Omega})$ \\
\hline 100 & 10.1 \\
\hline 150 & 1.3 \\
\hline 200 & 0.5 \\
\hline 250 & 0.4 \\
\hline
\end{tabular}

\section{CONCLUSION}

In this report, the AgNPs were synthesized by chemical reduction method by using the ultrasonic probe in EG. The AgNPs existance was convinced with the surface plasmon resonance shown in 400-420 $\mathrm{nm}$ region of $\mathrm{UV}-\mathrm{Vis}$ spectrum. TheTEM observation also confirmed that the particles were spherical and had diameter smaller than the nozzles of the printer. Furthermore, the influence of the heat treatment on the conductive pattern was investigated. The heat treatment from $200^{\circ} \mathrm{C}$ to $250^{\circ} \mathrm{C}$ was suitable temperature to obtain the sintered AgNPs to form the conductive layer. The resistivity measurements proved that the printed patterns had low resistivity. This conductive ink solution can be applied for fabrication of high efficiency electronic application by inkjet printing.

\section{Acknowledgments}

This research is funded by FIRST Central Project Management Unit Grant Agreement No.: 09/FIRST/2a/INT.

\section{REFERENCES}

1. Daniel M C and Astruc D 2004 Chem. Re. 104293

2. Bogunia-Kubik $\mathrm{K}$ and Sugisaka M 2002 Biosystems 65123

3. Zharov V P, Kim J W, Curiel D T and Everts M 2005 Nanomedicine: NBM. 1326 
4. Fahmy A, Eisa W H, Yosef M and Hassan A 2016, J. Spectroscopy, Article ID 7489536

5. Singh A, Jain D, Upadhyay M K, Khandelwal N and Verma H N 2010 Dig. J. Nanomater. Bios. 5 483

6. Durán N, Marcato P D, De Souza G I H, Alver O L and Waposito E 2007 J. Biomedical. Nanotechnol. 3203

7. Ganesharajah M and Koneswaran M 2018 Int. J. Nano. Med. Eng. 363

8. Wu Y, Liao L-D, Pan H-C, He L, Lin C-T and Tan M C 2017 RSC Adv. 729702

9. Kotthaus S, Gunther B H, Hang R and Schafer H 1997 IEEE Trans. Compon. Packaging. Technol. 2015

10. Wang F, Mao P and He H 2016 Sci. Rep. 621398

11. Ghosale A, Shankar R, Ganesan V and Shrivas K 2016 Electrochim. Acta 209511

12. Cho M S, Choi W H, Kim S G, Kim I H and Lee Y 2010 J. Nanosci. Nanotechnol. 106888

13. Park B K, Kim D, Jeong S, Moon J and Kim J S 2007 Thin Solid Films 5157706

14. Liu Q, Zhou D, Nishio K, Ichino R and Okido M 2010 Material Transaction 511386

15. Navaladian S, Viswanathan B, Varadarajan $T \mathrm{~K}$ and Viswanath R P 2008 Nanotechnology 19 045603

16. Niasari M S and Fereshteh Z 2009 Polyhedron 28 126

17. Abid J P, Wark A W, Brevet P F and Girault H H 2002 Chem. Commun. 7792

18. Schwarz F, Thorwarth G and Stritzker B 2009 Solid State Sci. 111819

19. Iravani S, Korbekandi H, Mirmohammadi S V and Zolfaghari B 2014 Res. Pharm. Sci. 9385

20. Oliveira M M, Ugarte D, Zanchet D and Zarbin A J G 2005 J. Colloid Interface Sci. 292429

21. Ajitha B, Kumar Reddy Y A, Reddy P S, Jeon H-J and Ahn C W 2016 RCS Adv. 636171

22. Evanoff D D Jr and George C 2005 Chem. Phys. Chem. 61221 